\title{
CT and MRI in Suspected Ischemic Heart Disease
}

\author{
Albert de Roos and Konstantin Nikolaou
}

\section{Learning Objectives}

- To understand basic approaches of CT and MRI in ischemic heart disease.

- To learn systematic approach to diagnose ischemic heart disease.

- To learn about differential diagnoses shown by CT and MRI.

\subsection{Introduction}

CT and MRI have made enormous strides over the past decades becoming established imaging techniques in patients with suspected ischemic heart disease. The impact of these imaging techniques cannot be overestimated in the diagnosis and prognostication of patients with ischemic heart disease. As ischemic heart disease is highly prevalent, any contribution to improving the diagnosis and work-up of these patients by noninvasive imaging techniques has direct implications to more cost-effective diagnosis and therapy. For a long time, the mainstay in the diagnosis and treatment of ischemic heart disease was conventional x-ray angiography and echocardiography. The recognition of limitations inherent to these well-established diagnostic methods has led to a surge of interest in cardiovascular imaging by CT and MRI. In addition, nuclear medicine imaging is a well-established imaging technology for myocardial perfusion assessment in patients with suspected ischemic heart disease. The strength of this

A. de Roos $(\bowtie)$

Department of Radiology, Leiden University Medical Center,

Leiden, South Holland, The Netherlands

e-mail: a.de_roos@lumc.nl

K. Nikolaou

Department of Diagnostic and Interventional Radiology, University

Hospitals Tubingen, Tubingen, Germany

e-mail: Konstantin.Nikolaou@med.uni-tuebingen.de imaging technology is the wealth of outcome data for patient management, but CT and MRI have also made big strides to pose as an alternative for myocardial perfusion imaging as well as other approaches to assess the functional significance of coronary artery disease.

CT and MRI have their own strengths and weaknesses to provide diagnostic information in the work-up of patients with suspected ischemic heart disease. The main breakthrough was coronary CT angiography providing unparalleled high-quality noninvasive visualization of coronary artery disease, now firmly established as the preferred tool to exclude coronary artery disease in patients with intermediate likelihood for coronary disease based on their clinical risk profile. The main limitation of CT was the lack of functional information to estimate the significance of a stenosis as a flow-limiting lesion that may cause myocardial ischemia. Nowadays CT may provide this crucial information for patient management by rest/stress CT perfusion and noninvasive estimation of the fractional flow reserve (CT-FFR). The integration of diagnosing coronary artery anatomy (stenosis) in conjunction with functional assessment (ischemia) provides a complete tool for clinical decision-making (indication for percutaneous coronary intervention or bypass surgery). In contrast, MRI has lacked behind to $\mathrm{CT}$ as a robust imaging tool for noninvasive coronary artery imaging. The main strengths of cardiac MRI are the unsurpassed highquality images of cardiac anatomy and global left ventricular function and a wide armamentarium for myocardial tissue characterization. For example, MRI late gadolinium enhancement (LGE) started over 30 years ago and took a relative long time before becoming established as a unique noninvasive tool to assess the transmural extent of a myocardial scar with great clinical implications. The combined use of cine MRI and LGE is clinically highly relevant to characterize ischemic heart disease and to differentiate ischemic from nonischemic heart disease. Cine MRI is unsurpassed in accuracy for precisely measuring structure (wall thickness) and function (ejection fraction). Next to LGE, a number of other 
MRI techniques have been established for myocardial tissue characterization that provide additional information on acute versus chronic scar (T2 mapping, STIR) and diffuse myocardial fibrosis (T1 mapping, extracellular volume or ECV). Combining these MRI techniques for tissue characterization has some similarity with how a pathologist may combine different staining techniques for tissue characterization to come to a final diagnosis. MRI myocardial tissue "staining" has been established as an invaluable tool for characterizing myocardial disease in patients with suspected ischemic heart disease as well as in patients with unexplained heart failure to differentiate ischemic heart disease from the large number of nonischemic cardiomyopathies (nonischemic dilated cardiomyopathy, hypertrophic cardiomyopathy, amyloid, other storage diseases, and alike).

Patients are referred to cardiac or chest CT and MRI by various medical disciplines (e.g., cardiology, internal medicine, emergency department) with different clinical questions and scenarios. For example, many referrals for evaluating acute chest pain come from the emergency department ("rule out pulmonary embolism"). The current high volume of pulmonary embolism referrals may lead to a number of unexpected findings on chest CT, as the yield of this referral for a positive diagnosis of pulmonary embolism is quite low and the clinical work-up of these patients may be suboptimal. The high-quality CT images of today, even if ungated, provide good diagnostic information on cardiac anatomy and coronary disease. So, coronary artery disease is becoming an "incidental finding" on routine chest CT requested for other indications. Radiologists are now in a central and responsible position to diagnose or rule out not only pulmonary embolism in acute chest pain evaluation but also be prepared to diagnose the extensive differential diagnosis (over 30 differential diagnoses) of a patient presenting with acute chest pain (main causes are life-threatening acute coronary occlusion, aortic dissection, and pulmonary embolism).

In this course we will discuss the role of CT and MRI in patients with suspected ischemic heart disease. First, the basic aspects of the pathophysiology (ischemic cascade) and technology will be shortly summarized. Secondly, the specific utility of CT and MRI in suspected ischemic heart disease will be illustrated using patient examples. The patient presentations will also consider a stepwise approach on how to differentiate ischemic from nonischemic cardiomyopathies and the differential diagnosis of acute chest pain.

\section{Key Point}

- LGE is helpful MRI technique to differentiate ischemic from nonischemic cardiomyopathy.

\subsection{Cardiac MRI}

\subsubsection{MRI Techniques}

Myocardial function is typically evaluated using cine balanced steady-state free precession (bSSFP) imaging, providing optimal contrast between the blood pool (bright signal) and myocardium (intermediate-low signal). Cine MRI is used to assess ventricular function and valvular function in various orientations (transverse, angulated). As a starting point, the transverse cine images provide a global impression on left ventricular function, regional function (scar, aneurysm), and valvular function (stenosis jet, regurgitation jet, valve morphology). Short-axis cine images are routinely applied for calculation of ventricular volumes (ejection fraction is very important for clinical decision-making and prognostication). LGE-MRI is the reference standard for imaging myocardial scar. Based on inversion recovery methods, contrast is created between normal (dark, gadolinium excluded from intact cells) myocardium and scar (white due to gadolinium accumulation). Both acute and chronic infarct may display LGE. Acute infarcts show LGE due to cell membrane breakdown, whereas chronic collagen scar has little cellular volume and larger volume of distribution and therefore accumulates gadolinium. A particular strength of LGE is visualization of very small scars as well as the transmural extent of necrosis (starts at the subendocardium and may progress through the ventricular wall as a wave front with longer durations of coronary occlusion). Myocardial signal intensity depends on the $\mathrm{T} 1, \mathrm{~T} 2, \mathrm{~T} 2 *$, and proton density of the tissue. All these parameters are actively explored for better characterizing the severity of myocardial injury. Quantitative maps of myocardial T1, T2, T2*, and extracellular volume (ECV) are attractive alternative methods for better tissue characterization. Quantitative methods for characterizing myocardial tissue based on parametric mapping of T1 and T2 have been explored as an objective tool of detecting and quantifying focal as well as global myocardial tissue alterations. In combination with extracellular contrast agent (gadolinium) injection, T1 mapping can also estimate the extracellular volume (ECV) fraction. Non-contrast enhanced, native T1 mapping and ECV mapping are explored as a diagnostic tool in both acute and chronic myocardial infarction and may be used to assess the area at risk (edema). Many other disease processes in the myocardium may result in elevated (e.g., myocarditis, amyloidosis) or decreased (e.g., Fabry disease, high iron content) native T1 values. Native T2 mapping can detect edema in myocarditis, area at risk, and acute myocardial infarction. Technical details of pulse sequences, acquisition protocols, scanner adjustments, artifacts, image processing (windowing) and other confounders are affecting and complicating reliable determination of parametric maps. Well-controlled and optimized techniques 
are crucial to improve accuracy (saturation methods), precision (inversion methods), and reproducibility of the use of parametric imaging. The acquisition technique combines an inversion or saturation pulse to pertubate T1, sampling of the relaxation curve, and a model to fit the sampled curve and extract the myocardial T1 value. Initial methods for pixelwise parametric T1 mapping are MOLLI and shMOLLI (modified Look-Locker inversion and shortened breath-hold modified Look-Locker inversion, respectively) sequences. Inversion methods (e.g., MOLLI, shMOLLI), saturation methods (e.g., SASHA, saturation recovery single-shot acquisition), and combined inversion and saturation methods (e.g., SAPPHIRE, saturation pulse prepared heart-rateindependent inversion recovery) are explored to optimize the reliability of acquisition schemes. Motion artifacts have been reduced by schemes that allow high-resolution imaging at the same cardiac and respiratory phase. The ECV in the myocardium is estimated from the concentration of extracellular contrast agent in the myocardium relative to the blood in a dynamic state. The formula for calculation of ECV incorporates hematocrit (determined for each individual from blood sample or synthetic Ht, reflecting blood volume of distribution) and change in relaxation rate between precontrast and postcontrast (proportional to gadolinium concentration, $15 \mathrm{~min}$ after bolus injection). Quantitative T2 mapping is commonly performed using single-shot T2-prepared SSFP approach, although other approaches are available. For example, images may be acquired at three echo times, and after monoexponential curve fitting, a pixelwise T2 map is produced.

The initial area at risk after coronary occlusion (before necrosis develops) may be visualized as a bright region by using T2-weighted MRI techniques. Multiple MRI techniques are available to provide optimal T2 contrast. T2-weighted imaging is also helpful to differentiate acute (white) from chronic scar (dark), as both may show similar LGE. Shortly after coronary occlusion edema may develop in the area at risk (the myocardium dependent on that coronary artery) and may be shown as a bright region on T2-weighted imaging. Initially dark-blood T2-weighted sequences were used for defining the presumed edema in the area at risk. However, when limitations and artifacts of these techniques raised some controversy on their utility, T2 mapping, T1 mapping, early gadolinium enhancement, and contrast-enhanced bSSFP cine MRI were proposed as alternative methods to visualize the area at risk. The area at risk appears bright on T2-weighted imaging (long T2) or dark on T1-weighted imaging (long T1) and in the bright range of the color scale on quantitative T1 and T2 maps due to long T1 and long T2. The endocardial surface area (approximation of the wave front) method of acute infarction has also been used to estimate the area at risk. The ischemic cascade may further progress with necrosis starting at the subendocardial level and depending on duration of occlusion may progress throughout the entire wall (transmural, full thickness infarct). LGE MRI may show the transmural extent of the infarct to best advantage. In large infarcts with extensive necrosis, a central area of no enhancement may be seen (microvascular obstruction). T2* imaging is useful to detect intramyocardial hemorrhage due to susceptibility for breakdown products of hemoglobin. Intramyocardial hemorrhage is considered as an additional marker of more severe myocardial injury, like microvascular obstruction. A number of other complications may be observed on cardiac MRI in ischemic heart disease (aneurysm, thrombus, contained rupture).

\section{Key Point}

- Acute as well as chronic myocardial infarction show LGE. T2-weighted MRI is helpful to differentiate acute from chronic myocardial injury.

\subsection{Myocardial Infarction}

Acute coronary occlusion leads to myocardial necrosis in the distribution area of the culprit coronary artery. Occlusion of the left anterior descending area will cause necrosis in the anterolateral wall, occlusion of the right coronary artery most commonly involves the inferoseptal wall, and occlusion of the circumflex coronary artery will involve the lateral wall. Inversely, recognizing scar in these locations may help to suspect the culprit artery. Ischemic heart disease can be suspected by regional abnormalities in the distribution area of individual coronary arteries. LGE is currently the highest-resolution method for detecting acute myocardial infarction with very high sensitivity, even to demonstrate microinfarcts (e.g., embolic infarction in patients undergoing percutaneous coronary intervention). The size of the region of hyperenhancement is a clinically validated measurement of infarct size, correlating with the degree of elevations of cardiac enzymes and other markers of necrosis. Acute myocardial infarcts will show LGE with variable transmurality and sometimes central microvascular obstruction (larger infarcts, worse prognosis). Microvascular obstruction and related no-reflow phenomenon can prevent reperfusion of the core of the infarct after reperfusion therapy and indicates more severe ischemia. Note the transmural extent of the infarct (grading more or less than $50 \%$ of wall thickness, indicating residual viability if less than $50 \%$ ). Viability assessment based on grading transmurality of the infarct may help to guide revascularization options (percutaneous intervention or bypass surgery). When analyzing patients with heart failure, the first step is to suspect regional abnormalities in the myocardium (e.g., a scar or 
wall thinning in the anterior wall is likely due to occlusion of the left anterior descending (LAD) artery). Wall thinning indicates the presence of long-standing scar (i.e., chronic infarct). For example, coronary CT angiography may show occlusion of the LAD and MRI may show LGE of chronic scar in the anterior wall. However, recanalization of a coronary artery may occur after myocardial infarction; therefore not all patients with a previous infarct will reveal occlusive coronary artery disease. Complications of previous infarcts include a left ventricular aneurysm (wide connection, involving all wall layers, true aneurysm), pseudoaneurysm (narrow neck, pericardial enhancement due to contained rupture in pericardial sack, predilection for inferior wall, large size), and thrombus adjacent to scar (best shown on LGE imaging). Thrombus is a common complication after myocardial infarction and may be missed by echocardiography, especially in apical location. LGE imaging is helpful to detect both acute and chronic infarction. T2-weighted imaging is used to differentiate acute versus chronic infarction. CT may also show the infarct as a perfusion defect and sometimes late iodine enhancement may reveal the infarct, although LGE MRI is better suited to demonstrate late enhancement with greater conspicuity. It is important to be aware of "incidental perfusion defects" in the myocardium when evaluating chest CT in patients with unexplained chest pain. In the scenario of acute chest pain, look for the presence and extent of coronary calcifications as a marker of coronary atherosclerosis (visual grading minor, moderate or severe works well as compared to the Agatston score). Coronary anomalies may be noted in young patients (coronary aneurysm in Kawasaki disease) or "malignant" course between the pulmonary artery and aorta (cause for ischemia and sudden death in young people). There are a number of anatomic variants of coronary anatomy that may have limited clinical significance (e.g., anomalous course of circumflex posterior to aorta, bridging of LAD).

\subsection{Heart Failure}

In patients presenting with heart failure the first question is whether coronary artery disease (previous infarcts) is the underlying problem. Coronary CT angiography and cardiac MRI are well suited to assess comprehensively the presence and extent of ischemic cardiomyopathy (see above). However, many patients with unexplained heart failure may suffer from a large number of other disease entities that may lead to heart failure. A common cause for heart failure is idiopathic, nonischemic dilated cardiomyopathy. The systematic analysis of CT and MRI images may help to clarify the diagnosis of nonischemic dilating cardiomyopathy. Coronary CT angiography will most commonly show normal coronary anatomy, although sometimes coexisting coro- nary disease may be noted. The overall appearance of the left ventricle indicates global dilation and diffuse wall thinning in patients with nonischemic dilating cardiomyopathy (in contrast to ischemic cardiomyopathy where we expect regional scar or wall thinning), although sometimes threevessel coronary artery disease may also result in this phenotype (hibernating left ventricle where bypass surgery can be advantageous to reverse global ischemia). Cardiac MRI using cine imaging will show the same appearance in a dynamic format. Cine MRI is very accurate to estimate the volumes and ejection fraction. The ejection fraction is a crucial parameter to decide for ICD placement. LGE will most commonly show no scar or LGE in patients with nonischemic dilating cardiomyopathy. In a minority of these patients, a scar in the middle of the ventricular wall can be shown (midwall scar is a trigger point for arrhythmias and may be an indication for preventive ICD placement).

Another common cause for heart failure is characterized by the hypertrophic phenotype. The hypertrophic phenotype has a number of underlying causes for myocardial thickening. The wall thickening can be local ("humps and bumps") in any location, most common in the septum in patients with genetically determined hypertrophic cardiomyopathy (the most common cause for sudden death in young people), but can also present as diffuse wall thickening. Simple explanations for left ventricular hypertrophy may be the presence of long-standing high blood pressure and outflow obstruction (e.g., aortic valve stenosis, bicuspid aortic valve). Sometimes physiological hypertrophy may occur in endurance athletes that may be reversible after stopping heavy exercise. After excluding these obvious causes for left ventricular hypertrophy, it is important to analyze cine MRI images and LGE images to narrow the differential diagnosis of the hypertrophic phenotype. Cardiac MRI is now playing a crucial role in systematically analyzing the potential underlying cause in unexplained heart failure presenting with the hypertrophic phenotype. The genetic hypertrophic phenotype is an important first consideration. Most commonly local hypertrophy will be recognized in the septum, but it may occur in any location and may even present with diffuse, global hypertrophy. There are a number of secondary helpful clues for this diagnosis on imaging. On cine imaging there may be a jet phenomenon in the outflow tract to the aorta (hypertrophic obstructive cardiomyopathy, exaggerated by the elongated anterior leaflet of the mitral valve, systolic anterior motion $(\mathrm{SAM}))$, secondary mitral regurgitation with enlargement of the left atrium is frequent, supernormal ejection fraction (hypercontractile left ventricle), and clefts in the myocardium. LGE is helpful to assess scar distributed in the myocardium in genetic hypertrophy (nonischemic pattern) and is a marker for arrhythmia risk. Cardiac amyloidosis is another disease that may present with the hypertrophic phenotype and is characterized by diffuse subendocardial LGE (not 
confined to a coronary artery distribution area). A number of other storage diseases may present with the hypertrophic phenotype (e.g., Fabry disease, typical LGE in posterolateral wall, sometimes focal hypertrophy, dilated aorta). Fabry disease is characterized by sphingolipid accumulation intracellularly (shown by native T1 mapping, ECV unchanged), sometimes complicated by diffuse or focal scar. Therapy is available for Fabry disease, so important to recognize and diagnose early. Inflammatory diseases (SLE, myocarditis, sarcoid) may present also with heart failure and the hypertrophic phenotype. Myocarditis may be recognized by subepicardial LGE in typical locations. A number of drugs may accumulate in the heart and present with heart failure and hypertrophy (e.g., amiodarone, hydroxychloroquine used in rheumatology, simulating Fabry disease).

\section{Key Point}

- MRI is a first-line modality to diagnose the underlying cause in patients with heart failure.

\subsection{Cardiac CT}

\subsubsection{CT Technique and Low-Dose CT Coronary Angiography}

Technical developments such as wide detectors, dual source configuration, low $\mathrm{kV}$ scanning and high-pitch acquisitions have allowed to establish coronary CT angiography (CCTA) as an imaging modality that provides high temporal and spatial resolution, for motion-free cardiac imaging and detailed visualization of coronary or myocardial pathology. With increasing utilization of coronary CT angiography, especially low-dose CCTA acquisitions are constantly improving, in order to combine CT's high anatomical resolution with short examination times while still maintaining very low radiation exposure. Specifically, high-pitch CT (pitch $>3$ ), low $\mathrm{kV}$ (as low as $70 \mathrm{kV}$ ), and iterative reconstruction algorithms have resulted in cardiovascular exams with radiation exposure of $<1 \mathrm{mSv}$ [1]. For a detailed visualization of the cardiac and coronary anatomy, ECGtriggered protocols are recommended, although it has also been demonstrated, that the detection of the coronary arteries is possible even in a non-ECG-triggered spiral mode with high temporal resolution [2]. Most CCTA cases today will be scanned in a prospective ECG-triggered sequential scan ("step-and-shoot mode") or, if a dual-source CT scanner is available, in high-pitch scan mode, whereas retrospective ECG-gating should be avoided or reserved to special cases (high heart rates, arrhythmia) due to potentially high radiation exposure.

\subsubsection{Stable Angina}

Consequently, CCTA has emerged as a noninvasive, robust, and well-established diagnostic tool for the assessment and evaluation of patients with known or suspected CAD and in stable angina pectoris. There is a large body of evidence for these indications, demonstrating a high diagnostic accuracy with a sensitivity ranging from 94 to $99 \%$ and a specificity of $64-83 \%$ for the detection of significant coronary stenoses (i.e., diameter stenoses $>50 \%$ in a morphological assessment) [3]. Clinically, with its high negative predictive value ranging from 97 to $99 \%$, CCTA represents a reliable diagnostic imaging tool to rule out obstructive CAD, especially in low- to intermediate-risk settings [3-6]. As such, largescale registries and prospective trials have shown that CCTA serves as a valuable alternative to invasive diagnostic testing in patients with stable angina [7-9] and low to intermediate pretest likelihood of CAD (e.g., asymptomatic, younger patients) and in those with less extensive coronary artery calcification, especially in patients who are unable to undergo stress testing for functional testing [6, 9]. An example of a negative coronary CT angiography in a relatively young female patient with suspected CAD is provided in Fig. 15.1. Furthermore, due to the ability of CCTA for visualization and assessment of coronary atherosclerotic plaque, three-dimensional vessel trajectories and anatomical features of the coronary vessel segments as well as detection of pathological coronary artery alterations such as calcifications or occlusions, CCTA is gaining recognition as a useful imaging tool in pre-procedural planning of cardiovascular interventions, providing improved success rates for revascularization and post-procedural outcome after percutaneous techniques [10]. Besides its diagnostic value, there is also an increasing role of CCTA to predict cardiovascular risk over time, as several follow-up studies report an excellent prognosis for patients negative at CCTA for any CAD or coronary atherosclerosis, whereas outcome in patients with obstructive and also nonobstructive CAD is substantially worse $[11,12]$.

\subsubsection{Acute Chest Pain}

The work-up of patients in the emergency room is complex and sometimes suboptimal, leading to a high number of unnecessary hospital admissions. Both CT and MRI have been successfully used as a gatekeeper to diagnose lifethreatening causes of acute chest pain (i.e., acute myocardial infarction, aortic dissection, and pulmonary embolism). In the setting of acute chest pain potentially caused by an acute coronary syndrome (ACS) or non-ST-elevation myocardial infarction (NSTEMI), there is strong evidence that CCTA improves the efficiency of clinical decision-making in the 
Fig. 15.1 Coronary CT of a 48-year-old female with exertional atypical chest pain. The CT reveals the absence of coronary atherosclerotic plaque or stenosis. (a) Axial slice demonstrating the left main coronary artery (asterisk) and the left anterior descending coronary artery (arrow head). (b) Origin of the right coronary artery (white arrow) and proximal segment of the left anterior descending coronary artery (arrow head) and left circumflex coronary artery (open arrow). (c) Volume rendered three-dimensional reconstruction showing the left anterior descending coronary artery as well as the branch of the right coronary artery and the circumflex coronary artery. $(\mathbf{d}-\mathbf{f})$ Multiplanar curved reconstructions of the right coronary artery $(\mathbf{d})$, the left anterior descending coronary artery (e), and the circumflex coronary artery (f) demonstrating the absence of coronary atherosclerotic plaque or coronary artery stenosis. $A A$ ascending aorta, $L A$ left atrium, $R V$ right ventricle
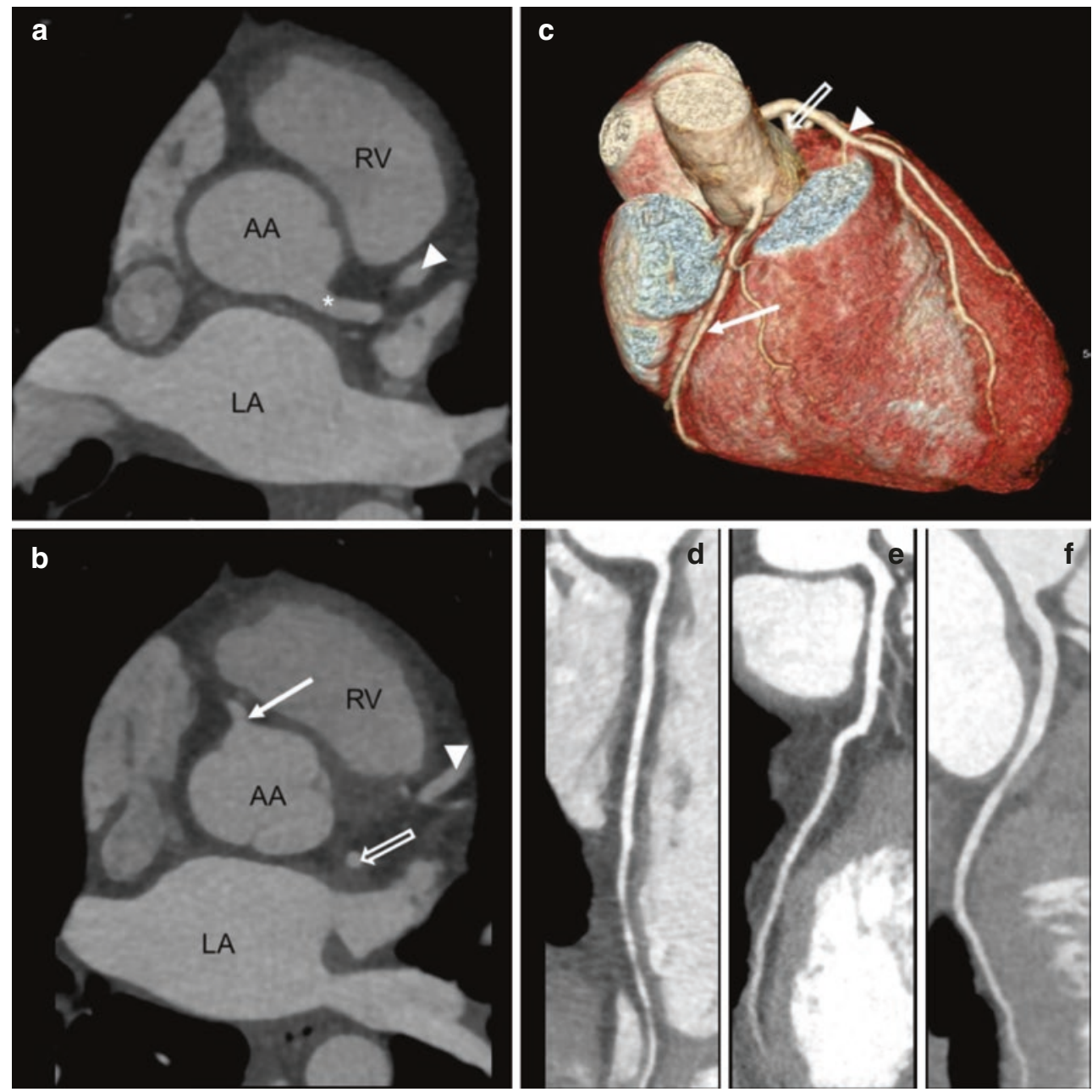

emergency department, resulting in a shorter length of hospitalization and reduced costs [13]. Several large randomized trials (CT-STAT, ACRIN-PA, ROMICAT II, and CT-COMPARE) compared coronary CT angiography to the current standard of care in patients with acute chest pain [13-16]. Their clinical implementations illustrated the reliability of a negative coronary $\mathrm{CT}$ angiography in identifying patients for discharge from the emergency department [17]. Here, coronary CT angiography allows for fast and safe triage of patients. In clinical practice, individuals demonstrating severe coronary stenosis are often directly referred to invasive catheter angiography, and patients with intermediate stenosis, high overall plaque burden, or equivocal findings typically undergo functional testing $[18,19]$.

\section{Key Point}

- Coronary CT angiography is a first-line modality for excluding coronary artery disease in patients with intermediate likelihood of disease.

\subsection{Functional Assessment of Coronary Artery Disease}

\subsubsection{CT-Based Fractional Flow Reserve (CT-FFR) and Imaging of Myocardial Perfusion}

Given its established value in morphologic imaging of CAD, research efforts over the last years have focused on providing information beyond the assessment of coronary stenosis and atherosclerotic vessel wall changes. To date, CT offers two different approaches to assess the functional relevance of a given stenosis: (A) myocardial perfusion imaging (which can be performed in a dynamic or static fashion) and (B) the calculation of a CT-based fractional flow reserve (CT-FFR). CT myocardial perfusion is based on the first-pass effect of iodinated contrast media through the myocardium during vasodilator stress (pharmacologically induced using regadenoson, adenosine, or dipyridamole). The study can either be performed by obtaining one single scan during maximum enhancement (also referred to as "static" or "snapshot/ 
single-shot" perfusion imaging) or by acquiring multiple scans during contrast passage (i.e., a "dynamic," timeresolved imaging of myocardial perfusion). The benefit of the "snapshot" protocol is the reduction of radiation exposure, whereas the "dynamic" protocol enables the absolute quantification of myocardial blood flow and volume. Perfusion defects as obtained by these techniques are identified as areas of hypo-attenuated myocardium compared to perfusion at baseline. While reversible malperfusion indicates ischemic myocardium, persistent perfusion defects are considered to represent myocardial scar tissue [20, 21]. The calculation of the CT-FFR using flow dynamics from coronary computed tomography angiography images is another noninvasive method for the assessment of flow dynamics [22]. This novel approach allows lesion-specific flow analysis throughout the coronary tree without additional imaging or vasodilator stress, i.e., this calculation is based on the original CCTA examination. The currently most widely used technique (known as FFRCT) uses a complex reconstruction algorithm to create a three-dimensional (3D) model of the coronary arteries and assess the left ventricular mass which is directly proportional to the total blood flow at rest. By combining the information of branch diameter from the 3D model and resting blood flow, it is possible to specifically calculate the blood flow for each coronary segment (see an example in Figs. 15.2 and 15.3). Recently, freely available and simplified approaches using a one-dimensional (1D) analysis (cFFR) are under investigation [23].

\section{Key Point}

- The presence or absence of flow limitation caused by a coronary artery stenosis can be estimated by computational fluid dynamics using the coronary CT dataset noninvasively on-site.

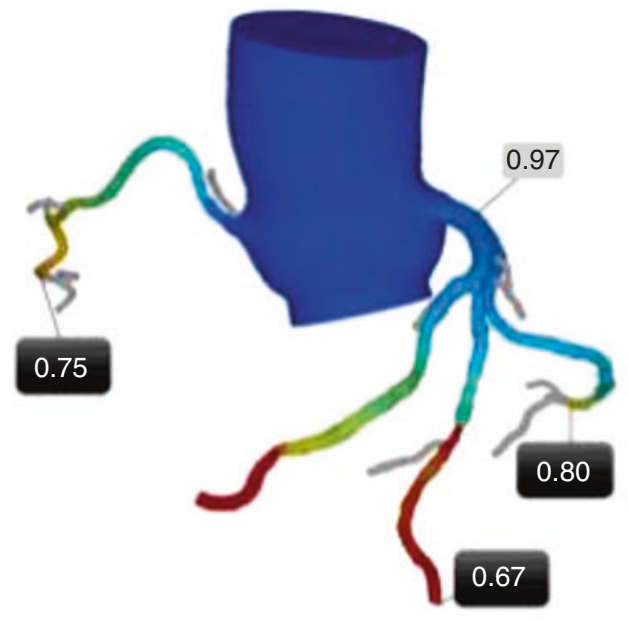

LAD

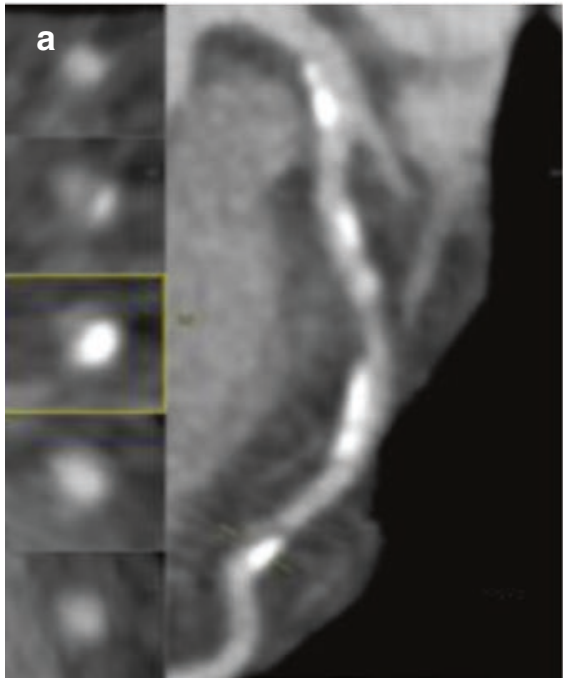

LCX

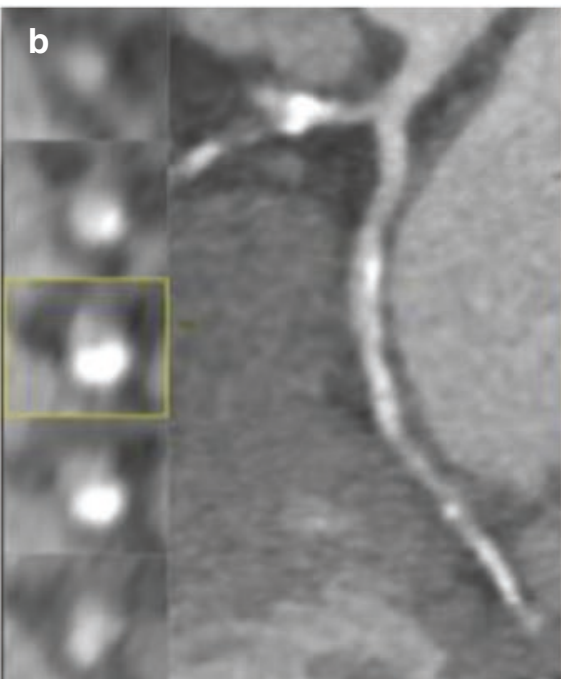

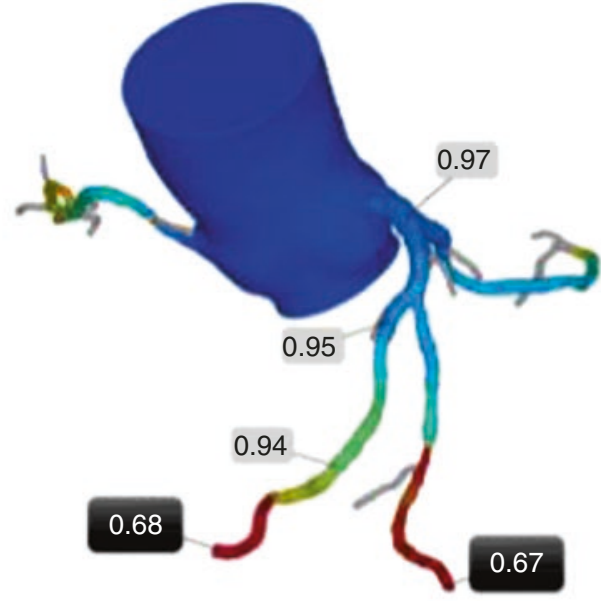

RCA

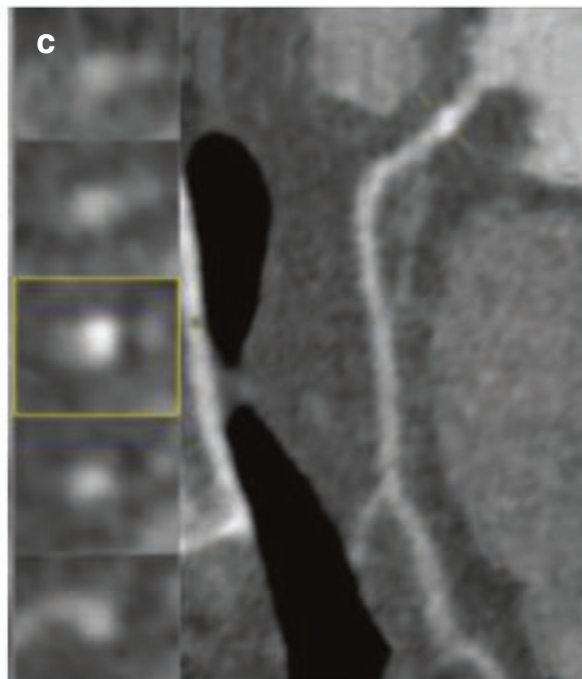

Fig. 15.2 Example of a CT-based derivation of fractional flow reserve model (CT-FFR, upper bar) and source coronary CT datasets demonstrating diffuse calcified and non-calcified plaques with hemodynamically relevant coronary stenosis present in the distal left anterior descending coronary artery (also A) and a large diagonal branch (red color-coding in the FFR model), whereas the right coronary artery and the left circumflex coronary artery ( $\mathrm{C}$ and $\mathrm{B}$, respectively) have diffuse coronary artery disease without hemodynamically relevant coronary lesions 


\subsection{Assessment of Atherosclerotic Plaque}

Given its high spatial resolution, CCTA offers the unique potential to noninvasively visualize, characterize, and quantify atherosclerotic plaque [24]. The notion of vulnerable plaques is a rather complex concept; however, specific highrisk plaque features can be identified on standard CCTA datasets. Several adverse features associated with vulnerable plaques have already been identified with CCTA; these include the presence of positive remodeling, low-attenuation plaque, spotty calcifications, and the napkin ring sign (see a typical low-attenuation plaque in Fig. 15.3) [25-28]. In contrast, larger calcification is more likely to be found in stable coronary lesions. Although the evidence is still very limited, detailed assessment of plaque morphology for detection of high-risk plaque features in patients presenting to the ED with acute chest pain but negative initial electrocardiogram and troponin may provide incremental diagnostic value, as the presence of high-risk plaque increases the likelihood of ACS, independent of significant CAD and clinical risk assessment, including age, sex, and number of cardiovascular risk factors [29]. Thus, identification of such high-risk plaques may thus improve risk estimates for the individual patient and may be of use in the selection of patients who benefit from revascularization.

\subsection{Summary}

Cardiac MR and CT imaging have seen an incredible speed of development over the past years. Today, both technique are readily and widely available and have reached a point of robustness while providing complex and complementary information at the same time, making both ever more important in clinical routine management of CAD patients.

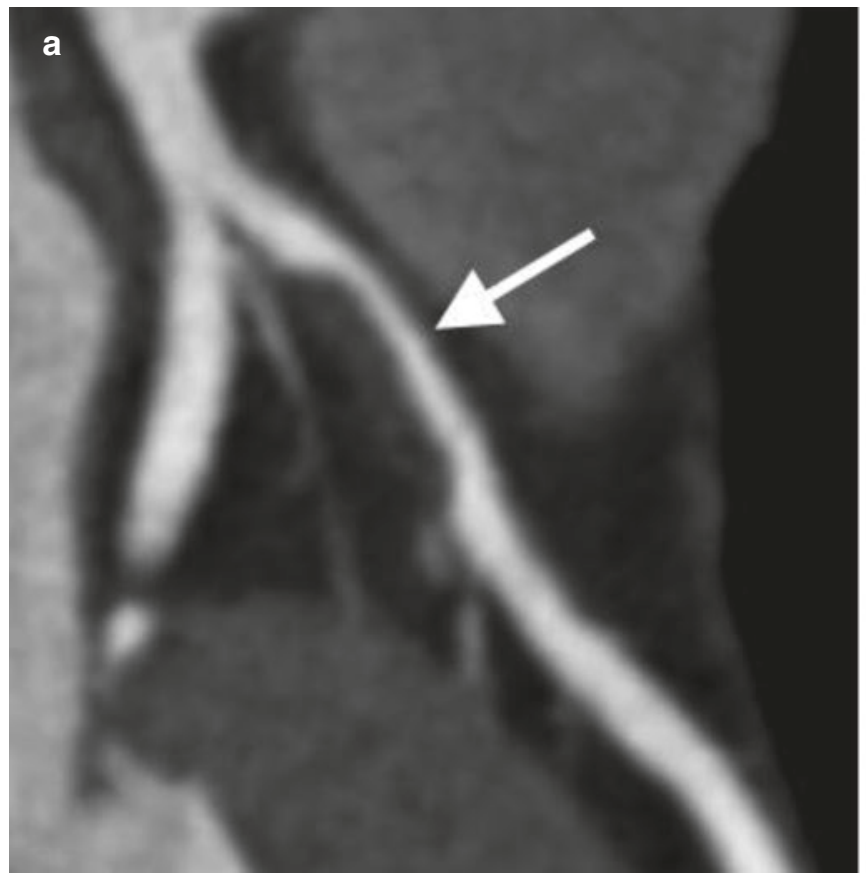

Fig. 15.3 Coronary CT of an atherosclerotic plaque in the LAD, demonstrating high-risk plaque features, including low-attenuation and positive remodeling in the proximal segment of the left anterior

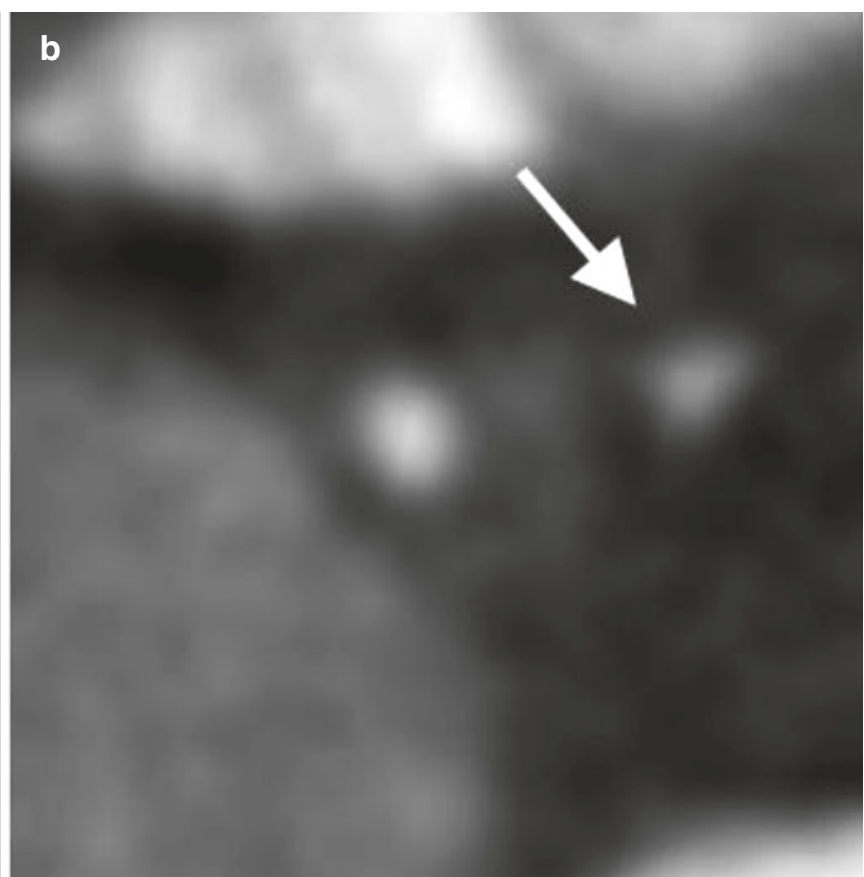

descending coronary artery. (a) curved multiplanar reformat and corresponding cross section of the vessel (b)

therefore for clinical decision-making in patients with ischemic heart disease.

- Coronary CT angiography is highly reliable for excluding coronary artery disease in patients with intermediate likelihood of disease.

- Functional assessment of an intermediate coronary stenosis requires ischemia testing by using myocardial perfusion or estimating fractional flow reserve by state-of-the-art CT techniques to guide treatment options. 


\section{References}

1. Rompel O, Glockler M, Janka R, et al. Third-generation dual-source $70-\mathrm{kVp}$ chest $\mathrm{CT}$ angiography with advanced iterative reconstruction in young children: image quality and radiation dose reduction Pediatr Radiol. 2016;46:462-72.

2. Bridoux A, Hutt A, Faivre JB, et al. Coronary artery visibility in free-breathing young children on non-gated chest CT: impact of temporal resolution. Pediatr Radiol. 2015;45:1761-70.

3. Budoff MJ, Dowe D, Jollis JG, et al. Diagnostic performance of 64-multidetector row coronary computed tomographic angiography for evaluation of coronary artery stenosis in individuals without known coronary artery disease: results from the prospective multicenter ACCURACY (Assessment by Coronary Computed Tomographic Angiography of Individuals Undergoing Invasive Coronary Angiography) trial. J Am Coll Cardiol. 2008;52:1724-32.

4. Hadamitzky M, Taubert S, Deseive S, et al. Prognostic value of coronary computed tomography angiography during 5 years of follow-up in patients with suspected coronary artery disease. Eur Heart J. 2013;34:3277-85.

5. Marwan M, Hausleiter J, Abbara S, et al. Multicenter evaluation of coronary dual-source CT angiography in patients with intermediate Risk of Coronary Artery Stenoses (MEDIC): study design and rationale. J Cardiovasc Comput Tomogr. 2014;8:183-8.

6. Meijboom WB, Meijs MF, Schuijf JD, et al. Diagnostic accuracy of 64-slice computed tomography coronary angiography: a prospective, multicenter, multivendor study. J Am Coll Cardiol. 2008;52:2135-44.

7. Douglas PS, Hoffmann U, Patel MR, et al. Outcomes of anatomical versus functional testing for coronary artery disease. N Engl J Med. 2015;372:1291-300.

8. Otaki Y, Arsanjani R, Gransar H, et al. What have we learned from CONFIRM? Prognostic implications from a prospective multicenter international observational cohort study of consecutive patients undergoing coronary computed tomographic angiography. J Nucl Cardiol. 2012;19:787-95.

9. Task Force M, Montalescot G, Sechtem U, et al. 2013 ESC guidelines on the management of stable coronary artery disease: the Task Force on the management of stable coronary artery disease of the European Society of Cardiology. Eur Heart J. 2013;34:2949-3003.

10. Opolski MP, Achenbach S. CT angiography for revascularization of CTO: crossing the borders of diagnosis and treatment. JACC Cardiovasc Imaging. 2015;8:846-58.

11. Hadamitzky M, Achenbach S, Al-Mallah M, et al. Optimized prognostic score for coronary computed tomographic angiography: results from the CONFIRM registry (COronary CT Angiography EvaluatioN For Clinical Outcomes: An InteRnational Multicenter Registry). J Am Coll Cardiol. 2013;62:468-76.

12. Dougoud S, Fuchs TA, Stehli J, et al. Prognostic value of coronary CT angiography on long-term follow-up of 6.9 years. Int J Cardiovasc Imaging. 2014;30:969-76.

13. Hoffmann U, Truong QA, Schoenfeld DA, et al. Coronary CT angiography versus standard evaluation in acute chest pain. N Engl J Med. 2012;367:299-308.

14. Goldstein JA, Chinnaiyan KM, Abidov A, et al. The CT-STAT (Coronary Computed Tomographic Angiography for Systematic Triage of Acute Chest Pain Patients to Treatment) trial. J Am Coll Cardiol. 2011;58:1414-22.
15. Litt HI, Gatsonis C, Snyder B, et al. CT angiography for safe discharge of patients with possible acute coronary syndromes. N Engl J Med. 2012;366:1393-403.

16. Hamilton-Craig C, Fifoot A, Hansen M, et al. Diagnostic performance and cost of CT angiography versus stress ECG-a randomized prospective study of suspected acute coronary syndrome chest pain in the emergency department (CT-COMPARE). Int J Cardiol. 2014; 177:867-73.

17. Cury RC, Abbara S, Achenbach S, et al. Coronary Artery Disease Reporting and Data System (CAD-RADS): An Expert Consensus Document of SCCT, ACR and NASCI: Endorsed by the ACC. JACC Cardiovasc Imaging. 2016;9:1099-113.

18. Meinel FG, Bayer RR 2nd, Zwerner PL, De Cecco CN, Schoepf UJ, Bamberg F. Coronary computed tomographic angiography in clinical practice: state of the art. Radiol Clin N Am. 2015;53:287-96.

19. Taylor AJ, Cerqueira M, Hodgson JM, et al. ACCF/SCCT/ ACR/AHA/ASE/ASNC/NASCI/SCAI/SCMR 2010 appropriate use criteria for cardiac computed tomography. A report of the American College of Cardiology Foundation Appropriate Use Criteria Task Force, the Society of Cardiovascular Computed Tomography, the American College of Radiology, the American Heart Association, the American Society of Echocardiography, the American Society of Nuclear Cardiology, the North American Society for Cardiovascular Imaging, the Society for Cardiovascular Angiography and Interventions, and the Society for Cardiovascular Magnetic Resonance. J Am Coll Cardiol 2010;56:1864-94.

20. George RT, Jerosch-Herold M, Silva C, et al. Quantification of myocardial perfusion using dynamic 64-detector computed tomography. Investig Radiol. 2007;42:815-22.

21. Dweck MR, Williams MC, Moss AJ, Newby DE, Fayad ZA. Computed tomography and cardiac magnetic resonance in ischemic heart disease. J Am Coll Cardiol. 2016;68:2201-16.

22. Pijls NH, De Bruyne B, Peels K, et al. Measurement of fractional flow reserve to assess the functional severity of coronary-artery stenoses. N Engl J Med. 1996;334:1703-8.

23. Nakanishi R, Budoff MJ. Noninvasive FFR derived from coronary $\mathrm{CT}$ angiography in the management of coronary artery disease: technology and clinical update. Vasc Health Risk Manag. 2016;12:269-78.

24. Nance JW Jr, Bamberg F, Schoepf UJ, et al. Coronary atherosclerosis in African American and white patients with acute chest pain: characterization with coronary CT angiography. Radiology. 2011;260:373-80.

25. Motoyama S, Kondo T, Sarai M, et al. Multislice computed tomographic characteristics of coronary lesions in acute coronary syndromes. J Am Coll Cardiol. 2007;50:319-26.

26. Maurovich-Horvat $\mathrm{P}$, Schlett CL, Alkadhi $\mathrm{H}$, et al. The napkin-ring sign indicates advanced atherosclerotic lesions in coronary CT angiography. J Am Coll Cardiol Img. 2012;5:1243-52.

27. Otsuka K, Fukuda S, Tanaka A, et al. Napkin-ring sign on coronary $\mathrm{CT}$ angiography for the prediction of acute coronary syndrome. J Am Coll Cardiol Img. 2013;6:448-57.

28. Motoyama S, Sarai M, Harigaya H, et al. Computed tomographic angiography characteristics of atherosclerotic plaques subsequently resulting in acute coronary syndrome. J Am Coll Cardiol. 2009;54:49-57.

29. Puchner SB, Liu T, Mayrhofer T, et al. High-risk plaque detected on coronary CT angiography predicts acute coronary syndromes independent of significant stenosis in acute chest pain: results from the ROMICAT-II trial. J Am Coll Cardiol. 2014;64:684-92.

Open Access This chapter is licensed under the terms of the Creative Commons Attribution 4.0 International License (http://creativecommons. org/licenses/by/4.0/), which permits use, sharing, adaptation, distribution and reproduction in any medium or format, as long as you give appropriate credit to the original author(s) and the source, provide a link to the Creative Commons license and indicate if changes were made.

The images or other third party material in this chapter are included in the chapter's Creative Commons license, unless indicated otherwise in a credit line to the material. If material is not included in the chapter's Creative Commons license and your intended use is not permitted by statutory regulation or exceeds the permitted use, you will need to obtain permission directly from the copyright holder. 